\title{
El efecto de las instituciones en el crecimiento económico de América Latina
}

\author{
Delfino Vargas Chanes, ${ }^{*}$ José Carlos González Nuñez**
}

Perfiles Latinoamericanos, 26(51)

2018 | pp. 329-349

DOI: $10.18504 / \mathrm{pl} 2651-013-2018$

\section{Resumen}

El objetivo del presente artículo es identificar las características relevantes de las instituciones que tienen un efecto sobre el crecimiento económico en los principales países de América Latina. Para ello se aplica un modelo multinivel, donde la variable dependiente es el producto per cápita real a precios de 2005, y las variables explicativas son los indicadores de gobernanza que estima el Banco Mundial. Los resultados muestran que las variables significativas que afectan el crecimiento económico son la calidad regulatoria y el control de la corrupción. Esto implica que se deben fortalecer las instituciones relacionadas con el Estado de derecho, la voz y rendición de cuentas, la estabilidad política y ausencia de violencia, y la efectividad gubernamental.

\begin{abstract}
The aim of this article is to identify relevant characteristics of institutions that have an effect on economic growth in selected Latin American countries. A multilevel model was used for this propose, the dependent variable is the real per capita GDP, and the explanatory variables are indicators of governance provided by the World Bank. The results show that the significant variables are regulatory quality and control of corruption. This implies the need to strengthen the institutions of the rule of law, voice and accountability, political stability and absence of violence, and government effectiveness.
\end{abstract}

Palabras clave: crecimiento económico, instituciones, gobernanza, calidad regulatoria, control de la corrupción y modelo multinivel.

Keywords: Economic growth, institutions, governance, regulatory quality, control of corruption and multilevel model.

* Doctor en Sociología por la Universidad Estatal de Iowa (Ph.D., 2000). Profesor del Programa Universitario de Estudios del Desarrollo de la unAm | dvchanes@unam.mx

** Doctor en Economía por la Universidad Nacional Autónoma de México y en Administración por la Universidad Anáhuac México Sur | josecarlos.gonzalez@anahuac.mx 


\section{Introducción}

E

xiste una creciente preocupación por estudiar el efecto de las instituciones en el crecimiento económico de los países de América Latina a fin de promover y mejorar la estructura institucional, así como adoptar políticas económicas que favorezcan ese crecimiento. Identificar las instituciones que son relevantes para tal propósito permite conocer a las que no lo son, con lo cual se pueden proponer medidas para su mejora y consolidación. Este artículo aborda el efecto de las instituciones de la gobernanza para el crecimiento económico de los países latinoamericanos. ${ }^{1}$

Con esa finalidad se utiliza un modelo lineal mixto o multinivel que ofrece un enfoque alternativo a la estimación tradicional de los modelos de regresión múltiple y tipo panel. Como la naturaleza de los datos se distingue por su estructura de anidamiento a lo largo del tiempo, el modelo multinivel resuelve la falta de independencia de las variables explicativas que miden la institucionalidad. ${ }^{2}$ Los datos que se han utilizado provienen de la base de datos del Banco Mundial, la cual es de libre disponibilidad, y se ha recurrido a las variables de gobernanza explicativas del crecimiento económico de 1996 a 2010, el cual se mide con el producto interno per cápita real a precios de dólares de 2005 (Weil, 2006).

La importancia de esta investigación radica en que son pocos los estudios cuantitativos cuyo propósito haya sido medir el efecto de las instituciones de gobernanza, las cuales han sido vistas como débiles en América Latina (Przeworski \& Curvale, 2006).

El artículo inicia con una semblanza de las diferentes teorías que explican el crecimiento económico de los países, en especial de la teoría institucional. Muchos consideran que esta es fundamental para explicar por qué algunos países han crecido más rápido que otros a pesar de contar con la misma dotación de recursos y que incluso los hay que, con menor dotación, han desplazado a aquellos con mejores condiciones geográficas (Rodrik \& Subramanian, 2003). El segundo apartado desglosa los datos y variables que representan a los principales países de América Latina y al periodo de análisis: de 1996 a 2010. El tercero se dedica a los modelos mixtos o multiniveles utilizados. En el cuarto se exponen los resultados de la modelación, la elección del mejor modelo en

1 Aquí se utiliza gobernanza según la define el Instituto del Banco Mundial, para el que la institucionalidad se representa con varios indicadores: voz y rendición de cuentas, estabilidad política/ausencia de violencia, efectividad gubernamental, calidad regulatoria, Estado de derecho y control de la corrupción (Kaufman, Kraay \& Mastruzzi, 2009).

2 La falta de independencia ocurre en el anidamiento natural de los datos. Es decir, cuando se llevan a cabo varias mediciones del mismo país a lo largo del tiempo. 
función de su poder explicativo y el ajuste de los datos. Mientras que la sección final está dedicada a las conclusiones en las que se identifica cuáles son las instituciones más relevantes y cuáles no.

\section{Marco teórico}

Para explicar el crecimiento económico hay tres grandes escuelas de pensamiento: 1) la teoría que asigna un papel preponderante a los recursos y el clima, es decir, la geografía; 2) la que destaca el papel del comercio internacional, 3) y la que se enfoca en las instituciones (Rodrik, Subramanian \& Trebbi, 2004).

La primera teoría se fundamenta en la disposición de los recursos naturales, de la mano de obra y del capital, entre otros elementos de producción. Ha sido una de las más recurrentes formas de explicar el crecimiento económico de los países y se focaliza en la acumulación de capital físico y humano y en el cambio tecnológico con su variante del crecimiento endógeno. La inversión en capital nuevo y la acumulación de capital humano elevan la productividad y contribuyen al crecimiento económico, pero el cambio tecnológico, es decir, el descubrimiento y aplicación de nuevas tecnologías permiten una contribución mayor (Weil, 2006).

La teoría económica de los recursos considera que se puede aumentar la riqueza de un país acumulando los factores de producción: mano de obra (horas de trabajo), capital físico y humano, y tecnología. Desde esta perspectiva, si un país quiere crecer debe ahorrar, con lo cual favorece la inversión que se traduce en incremento de capital y de producción, lo cual sigue el modelo de Solow (Todaro \& Smith, 2003). En este enfoque se sostiene que para obtener altas tasas de inversión y crecimiento se deben fomentar elevadas tasas de ahorro, y si el ahorro interno es insuficiente, un país podría recurrir al endeudamiento externo o facilitar la inversión para expandir el ahorro.

Sin embargo, muchos países en desarrollo, a pesar de haber elevado sus tasas de ahorro interno y obtener préstamos internacionales - como en América Latina durante los años setenta— no lograron mejorar sus tasas de crecimiento. $\mathrm{Al}$ contrario, en los ochenta sobrevino una crisis de deuda externa que originó una década perdida, debido al pobre crecimiento del ingreso per cápita que incluso se llegó a reducir en algunos países.

Tal escenario llevó a la necesidad de encontrar los determinantes fundamentales del crecimiento económico, lo que produjo y aumentó el interés por hallar explicaciones innovadoras. Fue así que surgió la teoría de la integración o comercio internacional y el estudio de las instituciones, la cual enfatiza en el papel del comercio internacional como conductor del cambio en la productividad. 
La segunda teoría enfatiza el papel del comercio internacional, como un conductor del cambio de productividad. De acuerdo con Rodrik, Subramanian \& Trebbi (2002), la integración al mercado mundial acelera la convergencia económica entre países pobres y ricos. Y según varios economistas (Frankel \& Romer, 1999; Sachs \& Warner, 1995), la teoría de comercio internacional de competencia perfecta considera que el comercio es una fuente subyacente de crecimiento que da la pauta para que las naciones se especialicen e incrementen así su productividad, lo cual mejora su nivel de bienestar.

Asimismo, la teoría de integración o de comercio afirma que cuando las empresas de un país compiten con las de otro se genera una especialización que conduce a economías de escala (a diferencia de cuando se quiere producir de todo), con lo que se reducen los precios, se motiva a producir bienes de mejor calidad —el cliente externo elige el bien que le dé la máxima utilidad combinando precio y calidad-, y se induce a la innovación permanente. De esta forma, las empresas adquieren una mayor disciplina que las obliga a comportarse eficientemente, lo que conduce a un ciclo virtuoso de inversión y crecimiento en los países que adoptan la política de integrarse al mercado disminuyendo las barreras al comercio internacional.

La apertura comercial de los años ochenta, profundizada luego en los noventa, ha logrado que las economías latinoamericanas se especialicen, con lo que ahora estas naciones producen y exportan bienes de mejor calidad y a precios competitivos; sin embargo, se requiere del desarrollo del capital humano para impulsar una mayor competitividad y producir bienes con más valor agregado.

La tercera teoría se concentra en las instituciones, en particular en el papel de los derechos de propiedad y el Estado de derecho. En esta perspectiva, lo más importante son las reglas del juego en una sociedad y su conducción para alcanzar un comportamiento económico deseable (North, 1993; Ayala, 2002; Rodrik et al., 2002). Es una postura que se asocia con lo que algunos han llamado infraestructura social (Hall \& Jones, 1999). Lo relevante, según Rodrik et al. (2002), es analizar por qué algunas sociedades administran e innovan más rápido que otras y por qué hay países que han crecido con más celeridad que otros pese a contar con los mismos recursos e incluso los hay con menos y aun así han desplazado a otros con mejores condiciones geográficas y de clima.

Rodrik et al. (2002) analizan cuál de las tres teorías esbozadas explica mejor el impacto en el desarrollo económico en el largo plazo y afirman que la tarea de demostrar causalidad es fácil para el determinismo geográfico. ${ }^{3}$ La geografía

3 Acemoglu \& Robinson (2012) también estudian diferentes teorías acerca del desarrollo y ofrecen una amplia discusión al respecto. Concluyen que muchas de ellas han fracasado en explicar el desarrollo y sugieren que las instituciones dan mayor claridad sobre el porqué los países fracasan. 
es una variable exógena y puede impactar directamente en el ingreso mediante sus efectos en la productividad agrícola y mortalidad e indirectamente a través de la distancia entre los mercados y su compleja integración, ya que esto último se traduce en costos de transportes y logística. Es decir, hay mayor ventaja competitiva de los mercados estrechamente vinculados respecto de los que no lo están o que por sus condiciones geográficas se dificulta la comunicación.

Los fundamentalistas del comercio y los institucionalistas encuentran mayor dificultad para demostrar el efecto de su variable independiente (comercio e instituciones) sobre el desarrollo económico. En cuanto al comercio, la variable más común es la apertura, es decir, el flujo de comercio relativo a la producción y su impacto sobre el crecimiento medido como ingreso per cápita. Respecto de los institucionalistas es necesario mostrar que una mejora en los derechos de propiedad o del Estado de derecho y otros aspectos institucionales son una variable independiente explicativa para el ingreso y no al revés, es decir, que las instituciones son consecuencia de ingresos altos.

En el trabajo de Rodrik et al. (2002), se ajusta un modelo econométrico con los datos de ochenta países y sus resultados demuestran que la calidad de las instituciones, medida por un indicador compuesto de varios elementos que captan la protección brindada a los derechos de propiedad y la solidez del Estado de derecho, es el único determinante positivo y significativo del nivel de ingreso. Cuando se neutralizan las instituciones, la variable integración pierde su efecto directo en el ingreso, mientras que la geografía reporta efectos débiles directos (Rodrik \& Subramanian, 2003).

Los institucionalistas sostienen que los trabajos se han enfocado en la importancia de los derechos de propiedad y en el cumplimiento de los contratos, lo que se denomina creadoras de mercado, puesto que en su ausencia estos no existen o funcionan mal (Rodrik et al., 2002). Pero el desarrollo económico a largo plazo exige además construir instituciones para mantener el ímpetu del desarrollo y facilitar así una distribución socialmente aceptable que las conserve.

Las instituciones ineludibles, de acuerdo con Rodrik et al. (2002), para garantizar un crecimiento a largo plazo serían: 1) las reguladoras del mercado que se ocupan de las externalidades, de las economías de escala y de la información asimétrica; 2) las estabilizadoras del mercado, que aseguran una estabilidad baja y evitan las crisis financieras, 3) y las legitimadoras de mercado, las cuales proporcionan seguro y protección social, se ocupan de la redistribución y controlan el conflicto.

Przeworski \& Curvale (2006), por su parte, analizan de qué manera las instituciones y los acontecimientos políticos fueron responsables de la brecha económica entre Estados Unidos y América Latina. Su postulado central es que las instituciones son la causa "primaria" del desarrollo económico, incluso 
más importante que las características del entorno natural, que las geográficas y que la oferta de factores y las tecnologías para utilizarlos. En suma, la calidad de las instituciones determina el crecimiento económico y prima frente a otras variables.

Es por ello que esta investigación analiza si las instituciones son relevantes para el crecimiento económico de los países de América Latina. Para esto se aplica un modelo que identifica a las instituciones que son importantes y las que no. El ulterior propósito es inducir a acciones de políticas que permitan su mejora.

\section{Datos y variables}

Para estudiar la relación entre la gobernanza y el crecimiento económico en América Latina se ha utilizado la información del Banco Mundial (вм) y la de su instituto sobre indicadores de desarrollo y gobernanza. La metodología para recuperar la información y construir los indicadores de la gobernanza se ha retomado de Kaufman, Kraay \& Zoido-Lobatón (1999). Se trata del método de componentes no observados, ${ }^{4}$ que usa los indicadores mundiales de gobernanza (WGI, por su sigla en inglés). El WGI se levanta en más de doscientos países y territorios y ha estimado seis dimensiones con mediciones periódicas a partir de 1996.

Los indicadores se basan en cientos de variables individuales subyacentes que se toman de una amplia diversidad de fuentes de datos. Estos reflejan los puntos de vista sobre la gobernanza de los países con base en la información que proviene de encuestas a nivel público, privado y de expertos de las ONG. El WGI también informa de márgenes de error que acompañan a la estimación de cada país y permite realizar comparaciones entre países en el tiempo. ${ }^{5}$ Son indicadores considerados como suficientes y consistentes.

El Instituto del Banco Mundial mide la gobernanza de los países a través de indicadores institucionales y diversos artículos donde se señala la fuerte relación entre el ingreso per cápita con la gobernanza, es decir, que una buena calidad de esta última redunda en una relación positiva con el crecimiento económico per cápita (Kaufman, Kraay \& Mastruzzi, 2009).

4 Para cada uno de los seis componentes no observables se mide a partir del puntaje de cada indicador $y_{j k}$ del país $j$ para el indicador $k$, que se obtiene a partir de una función lineal de gobernanza no observada en el país $j, g_{j}$ con un término de error $\varepsilon_{j k}$, que se resumen en la ecuación $y_{j k}=\alpha_{k}+\beta_{k}\left(g_{j}+\varepsilon_{j k}\right)$. Para mayores detalles véase Kaufmann, Kraay \& Mastruzzi (2010).

5 Los indicadores agregados y los datos desglosados de origen subyacente están disponibles en www .govindicators.org 
En consecuencia, las variables independientes son los indicadores de gobernanza de los países democráticos de América Latina, los cuales se agrupan en tres dimensiones que se capturan con dos indicadores:

a) El proceso por el cual los gobiernos, son seleccionados, monitoreados y reemplazados:

- Vozy rendición de cuentas $\left(\mathrm{X}_{1}\right)$ captura las percepciones de los ciudadanos de un país respecto de que sean capaces de participar en la selección de un gobierno. Incluye indicadores que miden aspectos del proceso político, libertades civiles y derechos políticos.

- Estabilidad politica y ausencia de violencia $\left(\mathrm{X}_{2}\right)$ captura la probabilidad de que el gobierno sea desestabilizado por medios violentos o inconstitucionales, incluyendo la violencia política y el terrorismo.

b) La capacidad del gobierno de efectivamente formular e implementar sus políticas:

- Efectividad gubernamental $\left(\mathrm{X}_{3}\right)$ captura las percepciones de la calidad de los servicios públicos, calidad de la burocracia, competencia de servidores públicos o civiles, independencia de los servidores públicos de las presiones políticas y credibilidad de los compromisos de las políticas del gobierno.

- Calidad regulatoria $\left(\mathrm{X}_{4}\right)$ captura la percepción de la incidencia de las políticas inadecuadas de mercado, tales como controles de precio o supervisión inadecuada de bancos, además de la percepción de gravamen impuesto por excesiva regulación en áreas como el comercio internacional y el desarrollo de negocios.

c) El respeto de los ciudadanos y del Estado para las instituciones que gobiernan la relación económica y social entre ambos:

- Estado de derecho $\left(\mathrm{X}_{5}\right)$ captura la percepción de la confianza de los agentes en las reglas de la sociedad, mide la calidad del cumplimiento de contratos, de la policía, y de las cortes, incluyendo la independencia judicial y la incidencia del crimen.

- Control de la corrupción $\left(\mathrm{X}_{6}\right)$ captura las percepciones acerca de si el poder público es ejercido para obtener beneficios privados, incluyendo la corrupción menor y en gran escala y la captura del Estado por las élites e intereses privados.

Los países incluidos en este análisis son Argentina, Bolivia, Brasil, Chile, Colombia, Ecuador, México, Paraguay, Perú, Uruguay y Venezuela. Naciones elegidas en función de la importancia de sus recursos y del valor de su producción en América Latina, el cual representa alrededor del 95\%. 
El periodo de análisis de los datos estadísticos que incorpora el modelo va de 1996 a 2014, esto es, los años de democracia. Justamente es después de 1996 que se dispone de información de las variables que miden la institucionalidad de la gobernanza democrática, lo cual permite el análisis y comprender si las instituciones son relevantes y explican el crecimiento económico de un país.

\section{Los modelos}

Para someter a prueba lo planteado por la teoría institucional se han desarrollado diversos modelos econométricos, los cuales se han aplicado con más frecuencia en la teoría de los recursos, dado que en ese ámbito existe información estadística económica suficiente. Sin embargo, son pocos los estudios cuantitativos que analizan el efecto de las instituciones de la gobernanza en el crecimiento económico, en especial en América Latina, donde los recursos de muchos de los países son similares. Es así que aquí se propone la aplicación de un modelo multinivel longitudinal en el que la variable dependiente es el producto interno per cápita real a dólares de 2005.

Aquí se ha utilizado un modelo multinivel longitudinal porque la naturaleza de los datos presenta una estructura de anidamiento a lo largo del tiempo. Es decir, que las variables de gobernanza de cada país se miden por varios ańos para cada país y eso explica la estructura de anidamiento al ser datos longitudinales.

Asimismo, los ciudadanos interaccionan con sus contextos sociales y son influidos por las características de los grupos y estos, a su vez, por los ciudadanos que los componen. Ciudadanos y grupos sociales entonces pueden conceptualizarse como un sistema multinivel. Estos se observan ampliamente en las ciencias sociales, donde se dispone de información acerca de los ciudadanos dentro de su contexto.

El modelo propuesto significa evitar la falta de independencia entre las observaciones (Raudenbush \& Bryk, 2002), además de que evalúa los efectos de las percepciones de la gobernanza en un cierto país a través del tiempo. Las herramientas estadísticas tradicionales (por ejemplo, la regresión de mínimos cuadrados ordinarios) ignoran la estructura multinivel de los datos y por ello conducen a conclusiones incorrectas (Snijders \& Bosker, 2012).

En seguida se presentan los modelos I, II y III a fin de comparar diferentes estrategias de análisis. También se incorpora un modelo nulo que se usa como base de comparación con los otros tres. El objetivo es seleccionar el modelo con mejor ajuste a los datos. 


\section{Modelo nulo}

Antes de proceder a incorporar las variables independientes se ha estimado un modelo nulo que incluye solo la variable dependiente donde los datos guardan una estructura de anidamiento en función de su variación a través del tiempo. El modelo es el que sigue.

$$
\begin{aligned}
& Y_{i j}=\pi_{o j}+\varepsilon_{i j} \\
& \pi_{0 j}=\beta_{00}+\mu_{o j}
\end{aligned}
$$

reemplazando la ecuación (2) en (1) se tiene,

$$
Y_{i j}=\beta_{00}+\left(\mu_{o j}+\varepsilon_{i j}\right)
$$

donde $Y_{i j}$, la variable dependiente es el logaritmo natural de la producción a nivel per cápita real de los países $i$ en el tiempo $j$. El modelo nulo permite estimar la producción per cápita de los países en el tiempo y el porcentaje de variación entre las naciones sin incluir ningún predictor en el modelo. De esta manera se puede comparar el efecto de las variables explicativas en la variable dependiente. Enseguida se presentan tres modelos alternativos para medir el efecto de las variables independientes de la gobernanza en el crecimiento económico de los países.

\section{Modelo I (sin inclusión del tiempo)}

El primer modelo considera solamente las variables explicativas y no incorpora la del tiempo. Se trata del siguiente modelo multinivel.

$$
\begin{aligned}
& Y_{i j}=\pi_{0 j}+\beta_{1} X_{1}+\beta_{2} X_{2}+\beta_{3} X_{3}+\beta_{4} X_{4}+\beta_{5} X_{5}+\beta_{6} X_{6}+\varepsilon_{i j} \\
& \pi_{o j}=\beta_{01}+u_{o j}
\end{aligned}
$$

sustituyendo la ecuación (5) en (4), se tiene el modelo mixto

$$
Y_{i j}=\beta_{0 j}+\beta_{1} X_{1}+\cdots+\beta_{6} X_{6}+\left(u_{0 j}+\varepsilon_{i j}\right)
$$

donde $\left(\mathrm{Y}_{\mathrm{ij}}\right)$, la variable dependiente, es el ingreso per cápita real del país $i$ en el tiempo $j$. Las variables independientes se representan por las $\left(\mathrm{X}_{1}\right)$, y son: voz y rendición de cuentas $\left(\mathrm{X}_{1}: \mathrm{voz}\right)$, estabilidad política y ausencia de violencia $\left(\mathrm{X}_{2}\right.$ :estabilidad), efectividad gubernamental ( $\mathrm{X}_{3}$ :efectividad), calidad regulatoria $\left(\mathrm{X}_{1}\right.$ :calidad), Estado de derecho $\left(\mathrm{X}_{5}:\right.$ ley) y control de la corrupción 
( $\mathrm{X}_{6}$ :corrupción). El término $\varepsilon_{i j}$ corresponde al error aleatorio del nivel-país y $u_{0 j}$ es el error aleatorio de la intersección.

\section{Modelo II (incluye el tiempo)}

A continuación se propone el modelo completo en el que se incorpora la variable tiempo y se busca estimar la tasa de cambio de la producción real a nivel per cápita del país a lo largo del tiempo. Las preguntas pertinentes son: ¿El tiempo es una variable relevante? ¿La tasa de cambio es positiva o negativa en el periodo estudiado? ¿Existen diferencias entre países? Las respuestas surgen al aplicar el modelo multinivel que tiene la ventaja de que no se necesita el mismo número de observaciones por individuo ni el mismo espaciamiento temporal entre las mediciones, ya que los modelos lineales multinivel son flexibles para incorporar el tiempo en el modelo (Snijders \& Bosker, 2012).

Este modelo II considera al tiempo como una variable explicativa y a él se le han agregado las seis variables independientes que operan el concepto de la institucionalidad denominada gobernanza y que abajo se aprecia.

$$
\begin{aligned}
& Y_{i j}=\pi_{0 j}+\pi_{1} X_{1}+\cdots+\pi_{6} X_{6}+\pi_{7 j} T_{j}+\varepsilon_{i j} \\
& \pi_{0 j}=\beta_{00}+\mu_{0 j} \\
& \pi_{7 j}=\beta_{70}+\mu_{7 j}
\end{aligned}
$$

Para construir el modelo se sustituye la ecuación (8) y (9) en (7) que da origen al modelo II mixto completo:

$$
Y_{i j}=\beta_{00}+\pi_{1} X_{1}+\cdots+\pi_{6} X_{6}+\beta_{7} T_{j}+\left(\mu_{0 j}+\mu_{7 j} T_{j}+\varepsilon_{i j}\right)
$$

donde $\left(\mathrm{Y}_{\mathrm{ij}}\right)$, la variable dependiente, es el ingreso per cápita real del país i en el tiempo j. En la ecuación (10), la parte que no está entre paréntesis se denomina efectos fijos, los cuales contienen las variables explicativas; la parte entre paréntesis contiene los efectos aleatorios.

El modelo II considera al tiempo como una variable independiente que representa la tasa de cambio, adicional a las variables de gobernanza. El anidamiento de los datos se hace en función de los países en el tiempo. Este modelo reconoce la variabilidad entre países a lo largo del tiempo.

\section{Modelo III (incluye interacciones con el tiempo)}

Este modelo contiene las variables predictoras significativas a partir del modelo II (Ec. 10) y busca evaluar las tendencias a lo largo del tiempo, en cuyo caso se 
incluye la interacción del tiempo con las variables explicativas de interés. Por ejemplo, si la variable predictora $\left(\mathrm{X}_{1}\right)$ es significativa, el modelo incorpora la interacción con el tiempo $\left(\mathrm{X}_{\mathrm{i}} \mathrm{T}_{\mathrm{j}}\right)$. El modelo mixto sería el siguiente:

$$
Y_{i j}=\beta_{00}+\pi_{1} X_{i}+\beta_{1} T_{j}+\beta_{2} X_{i} T_{j}+\left(\mu_{0 j}+\mu_{1 j} T_{j}+\varepsilon_{i j}\right)
$$

Con este modelo se prueba el efecto de la variable explicativa $\left(\mathrm{X}_{1}\right)$ a lo largo del tiempo en la variable dependiente, así como su tasa de cambio.

\section{Resultados}

\section{Elección del modelo}

Antes de proceder a la modelación e identificación de las instituciones que se consideran relevantes, primero se muestran las estadísticas descriptivas de las variables utilizadas en el modelo tal como se ilustra en el cuadro 1 .

\section{Cuadro 1. Estadísticas descriptivas de las variables de gobernanza $(n=209)$}

\begin{tabular}{lrrrr}
\hline Variable & Media & Desv. est. & \multicolumn{1}{c}{ Min } & Max \\
\hline Per cápita & 4604.40 & 2312.70 & 936.47 & 9853.53 \\
Voz y rendición de cuentas & 51.91 & 15.95 & 18.72 & 89.42 \\
Ausencia de violencia y estabilidad & 33.59 & 20.13 & 0.96 & 83.50 \\
Efectividad gubernamental & 47.22 & 21.37 & 9.48 & 87.80 \\
Calidad regulatoria & 49.96 & 23.99 & 2.87 & 96.57 \\
Estado de derecho & 37.30 & 22.44 & 0.48 & 89.47 \\
Control de la corrupción & 44.57 & 24.78 & 2.44 & 92.20 \\
\hline
\end{tabular}

Fuente: Elaboración propia con datos del Banco Mundial (1996-2014).

Las estadísticas descriptivas muestran un desempeño promedio de la institucionalidad de la gobernanza débil en América Latina, dado que en promedio solo una variable tiene un valor superior a 50, en una escala de 1 al 100, donde el mayor valor representa un mayor desempeño de acuerdo a las percepciones. En seguida se procede al análisis cuantitativo para identificar la institución de gobernanza relevante.

Para el modelo nulo (Ec. 3), el valor de la constante es $b_{0}=4,604.40$, que representa el promedio del ingreso per cápita real (cuadro 2). Ahora se ajusta el modelo I que incluye solo las variables explicativas. Los resultados se muestran en el cuadro 2 que adicionalmente contiene el modelo I completo y el reducido I que incorpora solo las variables que se consideran significativas (Ec. 6). 
Cuadro 2. Coeficientes estimados por máxima verosimilitud de los modelos multiniveles ajustados (Modelo I).

\begin{tabular}{|c|c|c|c|}
\hline Efectos fijos & Nulo & Completo I & Reducido I \\
\hline \multicolumn{4}{|l|}{ Variables a nivel de país } \\
\hline \multirow[t]{2}{*}{ Constante } & $4604.40^{\star \star \star}$ & $3083.52^{\star \star \star}$ & $2392.28^{\star \star \star}$ \\
\hline & $(663.21)$ & (797.96) & (721.36) \\
\hline \multirow[t]{2}{*}{ Voz y rendición de cuentas } & - & $38.52^{\star \star \star}$ & $33.74^{\star \star \star}$ \\
\hline & & (10.14) & (9.12) \\
\hline \multirow[t]{2}{*}{ Ausencia de violencia y estabilidad } & - & $16.61^{*}$ & $13.71 \dagger$ \\
\hline & & (7.71) & (7.96) \\
\hline \multirow[t]{2}{*}{ Efectividad gubernamental } & - & 1.37 & - \\
\hline & & (10.33) & \\
\hline \multirow[t]{2}{*}{ Calidad regulatoria } & - & $-34.38^{\star \star \star}$ & - \\
\hline & & $(7.03)$ & \\
\hline \multirow[t]{2}{*}{ Estado de derecho } & - & $23.06^{*}$ & - \\
\hline & & $(10.43)$ & \\
\hline \multirow[t]{2}{*}{ Control de la corrupción } & - & -5.51 & - \\
\hline & & (7.68) & \\
\hline \multicolumn{4}{|l|}{ Efectos aleatorios } \\
\hline Nivel individual & 2193.49 & 2002.71 & 1883.76 \\
\hline Nivel país & 715.25 & 620.09 & 678.83 \\
\hline ICC & $90.4 \%$ & $91.3 \%$ & $88.5 \%$ \\
\hline$-2 \times$ Log verosimilitud & 3397.58 & 3339.03 & 3373.55 \\
\hline Criterio del índice bayesiano (BIC) & 3401.09 & 3363.57 & 3384.06 \\
\hline
\end{tabular}

Nota: Los valores que se muestran en la tabla son estimaciones vía máxima verosimilitud con errores estándar entre paréntesis. $\dagger p<0.1 ;{ }^{\star} p<0.05 ;{ }^{\star \star} p<0.01 ;{ }^{\star \star \star} p<0.001$

Fuente: Elaboración propia con datos del Banco Mundial (1996-2014).

A partir del cuadro 2 se observa en la columna del modelo completo I las variables relacionadas con la efectividad gubernamental: no es significativa; la variable calidad regulatoria y control de la corrupción tienen signos negativos, lo cual es contraintuitivo y carece de validez teórica. Es decir, se esperan valores positivos que corresponden a que una mayor gobernanza tendrá un efecto positivo en la producción a nivel per cápita. La causa posible de este coeficiente negativo puede ser la alta colinealidad entre las variables independientes. Por ello se postulan varios modelos reducidos ${ }^{6}$ efectuándose diversas estimaciones, lo que da como resultado final el modelo reducido I, en el cual las mejores variables explicativas son voz y rendición de cuentas, y ausencia de violencia y estabilidad.

Sin embargo, el índice del criterio bayesiano (вІс, por sus siglas en inglés) es mayor que el índice correspondiente al modelo completo. Lo que sugiere un ajuste poco satisfactorio.

$6 \mathrm{Al}$ ajustar varios modelos se usa como criterio el que tenga el menor BIC. 
Con los resultados obtenidos y puesto que se dispone de un conjunto de datos para los once países para el periodo 1996-2014, se propone un modelo nuevo que incorpore al tiempo como variable independiente (Ec. 10). La razón de incluirlo responde a que se debe analizar el comportamiento de los once países de modo longitudinal y verificar si se tiene un modelo más robusto para explicar la variabilidad de la producción per cápita.

El modelo II (Ec. 10) se ajusta a los datos y los resultados se muestran en el cuadro 3. El modelo completo II muestra que algunas variables no son significativas como en voz y rendición de cuentas $(b=-9.539)$, ausencia de violencia y estabilidad, $(\mathrm{b}=-0.05)$, efectividad gubernamental $(\mathrm{b}=-3.91)$ y el Estado de derecho $(b=3.08)$. La variable tiempo es positiva y significativa $(b=132.81 ; p<0.001)$, por lo que se puede interpretar que por cada año los países tienen un incremento de 132.81 dólares, es decir, que en general el crecimiento es lento pero positivo.

Cuadro 3. Coeficientes estimados por máxima verosimilitud del modelo II que incluye el tiempo. (Modelo II)

\begin{tabular}{|c|c|c|}
\hline $\begin{array}{l}\text { Coeficientes estimados } \\
\text { Efectos fijos }\end{array}$ & Completo II & Reducido II \\
\hline \multicolumn{3}{|l|}{ Variables a nivel de país } \\
\hline \multirow[t]{2}{*}{ Constante } & $2108.83^{\star * \star}$ & $1,671.64^{\star \star}$ \\
\hline & $(619.11)$ & $(551.05)$ \\
\hline \multirow[t]{2}{*}{ Voz y rendición de cuentas } & -9.53 & -- \\
\hline & $(6.13)$ & \\
\hline \multirow[t]{2}{*}{ Ausencia de violencia y estabilidad } & -0.05 & -- \\
\hline & $(3.73)$ & \\
\hline \multirow[t]{2}{*}{ Efectividad gubernamental } & -3.91 & -- \\
\hline & $(4.67)$ & \\
\hline \multirow[t]{2}{*}{ Calidad regulatoria } & $32.25^{\star \star \star}$ & $31.73^{\star \star \star}$ \\
\hline & $(4.17)$ & $(3.05)$ \\
\hline \multirow[t]{2}{*}{ Estado de derecho } & 3.08 & -- \\
\hline & $(6.00)$ & \\
\hline \multirow[t]{2}{*}{ Control de la corrupción } & $5.76+$ & 3.93 \\
\hline & $(3.57)$ & (3.25) \\
\hline \multirow[t]{2}{*}{ Tiempo } & $132.81^{\star \star}$ & $130.43^{\star \star \star}$ \\
\hline & $(21.38)$ & $(21.27)$ \\
\hline \multicolumn{3}{|l|}{ Efectos aleatorios } \\
\hline Nivel individual & 1723.33 & 1675.11 \\
\hline Nivel país & 247.22 & 250.24 \\
\hline Pendiente & 69.248 & 69.13 \\
\hline ICC & $98.0 \%$ & $97.8 \%$ \\
\hline$-2 \times$ Log verosimilitud & 3012.93 & 3016.83 \\
\hline Criterio del índice bayesiano (BIC) & 3040.97 & 3030.85 \\
\hline
\end{tabular}

Nota: Los valores que se muestran en la tabla son estimaciones vía máxima verosimilitud con errores estándar en paréntesis; $+\mathrm{p}<0.1$; ${ }^{\star} \mathrm{p}<0.05$; ${ }^{\star \star} \mathrm{p}<0.01$; ${ }^{\star \star \star} \mathrm{p}<0.001$

Fuente: Elaboración propia con datos del Banco Mundial (1996-2014). 
Luego se procedió a estimar un modelo reducido eliminando las variables que no son significativas, lo que dio como resultado el modelo reducido II que contiene menos variables independientes (cuadro 3, columna "reducido II") y cuyas variables significativas fueron: calidad regulatoria $(b=31.73 ; \mathrm{p}<0.001)$ y el tiempo $(b=130.43 ; p<0.002)$, por último, se incluye la variable denominada control de la corrupción $(\mathrm{b}=3.93 ; \mathrm{p}=0.22)$. Esta última se considera relevante para analizar con el modelo III que incorpora la interacción con el tiempo. Adicionalmente, se observa que el BIC del modelo II reducido es el menor de los modelos previamente analizados. Igualmente, al comparar el modelo completo II con el reducido II, usando la prueba de razón de verosimilitudes (LRT, por sus siglas en inglés), se observa que el modelo reducido es mejor que el completo $(\mathrm{p}<0.05)$.

En el modelo reducido II del cuadro 3 se aprecia que un incremento de la calidad regulatoria en un punto porcentual tiene como consecuencia un incremento de producción per cápita en dólares de 31.73, con un nivel de confianza de $99 \%$. En lo referente al control de la corrupción, la mejora de un punto porcentual arroja un crecimiento de la producción per cápita de 3.93 dólares, con un nivel de confianza de 78\%; con relación al tiempo, el incremento de un año implica un crecimiento de la producción per cápita de 130.42 dólares por año, con un nivel de confianza de $99 \%$.

El modelo reducido II permite identificar tres variables con efecto en la producción per cápita de los países en el periodo de tiempo analizado. Es decir, la calidad regulatoria, el control de la corrupción y la tasa de cambio (tiempo). Este resultado, que se discute más adelante, muestra la debilidad de las instituciones de gobernanza en el crecimiento económico de los principales países de América Latina y la necesidad de fortalecerlas.

Finalmente, se ajusta el modelo reducido II aumentando la interacción del tiempo con las variables explicativas previamente identificadas y por ello se procede a multiplicar las dos variables de la institucionalidad de gobernanza con el tiempo. A este modelo se le denomina modelo III (Ec. 11). Los resultados se muestran en el cuadro 4.

En el modelo III las variables significativas son multiplicadas por el tiempo y la intención es conocer su efecto en la producción per cápita. Lo relevante es que la variable calidad regulatoria por el tiempo muestra un efecto negativo $(b=-1.93$; $\mathrm{p}<0.001)$. Es decir, que la calidad regulatoria de los países considerados en el periodo 1996-2014 ha ido empeorando, lo cual sugiere que la percepción de los ciudadanos es que el gobierno ha incorporado un mayor número de trámites burocráticos en el entorno empresarial durante el periodo analizado de lo que resulta una relación negativa.

En lo referente a la corrupción multiplicada por el tiempo es positiva y significativa $(b=2.03 ; \mathrm{p}<0.001)$, por lo cual se considera que cuando existe 
un mayor control de corrupción, se asocia con un crecimiento mayor en la producción.

Además se observa que la variable corrupción tiene un coeficiente negativo $(b=-14.02 ; \mathrm{p}<0.003)$. Esta variable no tiene necesariamente una interpretación práctica, la razón de haberla incorporado radica en que el modelo mixto contiene interacciones con el tiempo y se debe incorporar la variable independiente de interés en el mismo modelo. La interpretación solo tiene sentido para las variables que interactúan con el tiempo.

\section{Cuadro 4. Coeficientes estimados por máxima verosimilitud para el modelo III}

\begin{tabular}{lc}
\hline Coeficientes estimados & Reducido III \\
Efectos fijos & \\
\hline Variables a nivel de país & $1880.97^{\star \star \star}$ \\
\hline Constante & $(572.83)$ \\
Calidad regulatoria & $42.35^{\star \star \star}$ \\
& $(3.69)$ \\
Control de la corrupción & -14.02 \\
Tiempo & $(4.77)$ \\
Calidad regulatoria $\times$ tiempo & $137.07^{\star \star \star}$ \\
Control de corrupción $\times$ tiempo & $(24.80)$ \\
& $-1.93^{\star \star \star}$ \\
\hline Efectos aleatorios & $(0.39)$ \\
\hline Nivel individual & $2.03^{\star \star *}$ \\
Nivel país & $(0.44)$ \\
Pendiente & \\
\hline Criterio del índice bayesiano (BIC) & 1728.26 \\
\hline
\end{tabular}

Nota: Los valores que se muestran en la tabla son estimaciones vía máxima verosimilitud con errores estándar entre paréntesis. $+p<0.1 ;{ }^{*} p<0.05 ;{ }^{* \star} p<0.01 ;{ }^{\star \star *} p<0.001$

Fuente: Elaboración propia con datos del Banco Mundial (1996-2014).

Finalmente, se observa que el BIC para este modelo es igual a 3013.17, y es el menor índice de todos los modelos arriba ajustados. Las implicaciones de este hallazgo es que el modelo III es el mejor y la interpretación es que la calidad regulatoria, el control de la corrupción, así como sus interacciones con el tiempo explican mejor al crecimiento económico de los países estudiados durante el periodo 1996-2014. 
El coeficiente de correlación intraclase (ICC, por su sigla en inglés) en los modelos multinivel es fundamental porque evalúa el grado de correlación entre unidades de nivel micro tomadas al azar y que pertenecen a la misma unidad macro. En este caso, la unidad micro son las mediciones de las variables por cada país y el nivel macro corresponde al propio país en el tiempo. Snijders $\&$ Bosker (2012) justifican el uso del modelo multinivel al observar un ICC alto que significa que las unidades de estudio no son estrictamente independientes y es recomendable aplicar el mencionado modelo. Los resultados del coeficiente de correlación intraclase se exponen enseguida.

a) ICC para modelo nulo. Para estimar la variabilidad intrasujetos y entre países el modelo nulo permite estimar la correlación intraclase y los porcentajes de variación intra y entre países. La estimación del ICC es del 90.4\%, lo que indica que el país explica la variabilidad de la conducta de la producción a nivel per cápita, indicando que el modelo multinivel es útil para entender mejor el PIB per cápita.

b) ICC para el modelo II. El modelo II es más complejo que el anterior porque se le adicionan las variables de la estructura institucional de la gobernanza (variables micro, que representan la medición dentro del país), agregándole la variable macro del tiempo. El ICC del modelo reducido II es de $97.8 \%$, esto es, mayor a los modelos previamente ajustados, ya que explica que la diferencia de la producción per cápita se da por la característica contextual del país.

c) ICC para el modelo III. La correlación intraclase del modelo III, que señala la variabilidad de la producción per cápita, se explica en $98.1 \%$ por el contexto del país. Se puede afirmar que el modelo III es el mejor, dado que en este las variables institucionales explicativas son la calidad regulatoria, el control de corrupción y las interacciones de estas con el tiempo.

\section{Discusión de resultados}

Para los países de América Latina, los resultados acerca de que la calidad regulatoria es significativa para explicar el crecimiento económico son relevantes para los tomadores de decisiones en la política pública. Dichos resultados hablan de la habilidad del gobierno para implementar reglas y políticas que faciliten el correcto funcionamiento de los mercados (controles de precios o regulación excesiva) y que favorezcan e impulsen el desarrollo adecuado de los negocios y el comercio externo. 
En tal sentido, esto revela una relación directa con la competitividad y por tanto con el crecimiento económico, ya que la disminución en la cantidad de controles y regulaciones burocráticas relacionadas con la puesta en marcha de nuevos negocios es un factor clave para crear un adecuado ambiente empresarial y hay evidencia reciente que sugiere que es un mecanismo importante para la innovación, de acuerdo al estudio de Sachs \& Vial (2002), en el cual se analizan las facilidades o dificultades para iniciar nuevas actividades empresariales y se menciona que el desempeño institucional no es adecuado en América Latina. En su estudio, tales autores afirman que los empresarios opinan que esta es una de las mayores debilidades de la región:

En promedio la cantidad de procedimientos necesarios para poner en marcha un nuevo negocio en América Latina es más alto (13.5) que en el resto de los países (9.6); además, el tiempo necesario para completar todos los procedimientos es más largo (92.7 días en América Latina comparado con 57.9 días en el resto de los países); el porcentaje de tiempo que la alta gerencia le debe dedicar a los procedimientos burocráticos también es mayor (26\% comparado con $21.7 \%$ en otros países) (Sachs \& Vial, 2002: p. 19).

Estos resultados muestran que América Latina es una región con exceso de regulaciones y que esto preocupa a los que toman las decisiones para invertir. Los países que han logrado mejorar la calidad regulatoria logran un mayor impacto en la tasa de crecimiento.

En resumen, para los empresarios es relevante esta variable y los resultados estadísticos validan su importancia. A pesar de ello, ellos perciben que, en América Latina, en el tiempo analizado, la calidad regulatoria ha ido empeorando con mayores trámites, lo cual quiere decir que el crecimiento es menor debido a la reducción de inversión o la demora de esta por cumplir con normas y regulaciones.

Asimismo, otra variable relevante es el control de la corrupción, la cual refleja en qué medida los ciudadanos perciben ese fenómeno en su país. La corrupción, a grandes rasgos, se define como el uso abusivo del poder público para satisfacer intereses personales.

De acuerdo con Parker, Berthin, Michelle \& Mizrahi (2004), los países de América Latina comparten varias características que causan corrupción: a) leyes engorrosas, superposición de responsabilidades de diferentes organismos gubernamentales y confusión sobre leyes, b) procedimientos burocráticos y reglamentaciones complejas, $c$ ) debilidad de las instituciones de control y fiscalización, $d$ ) sistemas judiciales que no son eficientes e independientes, y $e$ ) controles sociales débiles y falta de voz, entre otros aspectos. 
La corrupción varía de un país a otro y oscila de normal a extendida y a sistémica (Parker et al., 2004). Se considera normal cuando es fácil identificar el problema, sancionar a los culpables y corregir lo que permitió la corrupción; sin embargo, una vez que se extiende y se hace sistémica, la probabilidad de detección y sanción se reduce y se crean incentivos para que aumente.

Si existe corrupción sistémica, las instituciones, es decir, las normas y reglas de comportamiento se han adaptado a una forma de operación corrupta y los actores públicos y privados se aprovechan de ello para su enriquecimiento personal. Así se generan incentivos para que los nuevos funcionarios, empresarios y nuevos actores se comporten de forma corrupta. En este caso, la corrupción causa grave daño a la estabilidad de las instituciones democráticas, erosiona el Estado de derecho y corroe el crecimiento económico y la competitividad (Parker et al., 2004).

Las consecuencias de la corrupción han comenzado a medirse y se han presentado pruebas empíricas de los costos económicos y sociales. En el informe de competitividad de América Latina 2001-2002 (World Economic Forum, 2002), se demuestra el vínculo entre crecimiento económico y corrupción. La corrupción sistémica obstaculiza la inversión nacional y extranjera, restringe el comercio, distorsiona el tamaño y composición de los gastos de gobierno, debilita el sistema financiero y fomenta la economía informal.

Para Sachs \& Vial (2002) existe una relación importante y significativa entre la corrupción y el crecimiento económico. Es decir, los países más corruptos presentan un menor crecimiento en relación con los menos corruptos. En virtud de ello han surgido diversos esfuerzos para controlar la corrupción, destacando la Convención Interamericana contra la Corrupción (CICC) de la Organización de Estados Americanos (OEA), en la cual los países se comprometen a implementar políticas destinadas a reducir la corrupción y aumentar la transparencia.

Otra parte de la discusión acerca del estancamiento en el desarrollo de los países es que este se detiene por la alta extracción de la renta y la presencia de los monopolios, además de la falta de controles y la ya citada corrupción (Acemoglu \& Robinson, 2012).

En este artículo se ha mostrado que controlar la corrupción tiene un efecto en el crecimiento económico de los países de América Latina. Lo importante de los resultados es que permiten apreciar que en el periodo de análisis se ha mejorado la percepción del control de la corrupción. No obstante, es insuficiente puesto que el efecto sigue siendo marginal o débil.

A modo de resumen, es posible señalar que los resultados muestran el poco efecto de las instituciones de gobernanza en el crecimiento económico de América Latina (cuadro 3), lo cual es más notable en el incremento del tiempo, lo que reafirma la percepción de un bajo desempeño institucional (cuadro 1). 
Y dada la interacción de las instituciones con el comportamiento (Sen, 2015: p. 15) se puede entender la conducta oportunista de los agentes - no cumplimiento de las normas o del Estado de derecho- y la obtención de beneficios personales indebidos que perjudican el crecimiento nacional.

La ausencia de un efecto significativo de las instituciones (voz y rendición de cuentas, ausencia de violencia y estabilidad, efectividad gubernamental y Estado de derecho) se explica en parte porque no se cumplen las características de protección de los derechos de propiedad de la mayoría de la población, de restricciones sobre las actuación de grupos de políticos y de élites - las cuales velan por sus propios intereses-, y de falta de oportunidades para grandes segmentos de la sociedad marginada (Acemoglu, 2003).

La mejora de las instituciones y el conseguir que estas produzcan un mayor efecto positivo en el crecimiento económico de los países será posible cuando se supere la visión de acumulación de capital y tecnología, es decir, cuando se facilite el acceso a los recursos financieros que incentivan la inversión y la formación de capital humano (Carrasco\& Castaño, 2012). Con ello será mejor el comportamiento de las personas y el de las instituciones, lo que apoyaría, a fin de cuentas, la generación de mayor riqueza, la redistribución del ingreso, y la expansión de libertades y capacidades individuales en América Latina.

\section{Conclusiones}

El presente estudio se ha enfocado al estudio del efecto de las instituciones de la gobernanza en el crecimiento económico de los países latinoamericanos. Con base en los resultados, se concluye que las instituciones significativas para este último fin son la calidad regulatoria y el control de la corrupción.

Para ello se utilizó un modelo multinivel longitudinal que permite resolver el problema de la multicolinealidad de las variables independientes y observar el efecto del tiempo, con ello se han estimado parámetros consistentes. Tradicionalmente se han utilizado modelos de regresión múltiple y tipo panel que no dan cuenta del efecto de anidamiento natural de los datos en el tiempo.

Con base en los resultados del periodo analizado (cuadro1) se muestra la debilidad institucional en América Latina, ya que los valores de la percepción promedio de los indicadores de gobernanza son menores de 55, en una escala de 1 al 100, donde el mayor valor representa un mejor desempeño.

Una recomendación de política pública es que en América Latina deben fortalecerse las instituciones relacionadas con la gobernanza, en especial el Estado de derecho y la ausencia de violencia y estabilidad, cuyos valores promedio son, de acuerdo a lo mostrado en este artículo, los más bajos en la región. 


\section{Referencias}

Acemoglu, D. (2003). Root Causes. A Historical Approach to Assessing the Role of Institutions in Economic Development. Finance and Development, 40(2), 27-30.

Acemoglu, D. \& Robinson, J. (2012). Por qué fracasan los paises. México: Deusto.

Ayala Espino, J. (2002). Fundamentos institucionales del mercado. México: UnAM.

Banco Mundial. (2011). World Development Indicators. Recuperado el 15 de julio de 2011, de http://data.worldbank.org/data-catalog/world-development-indicators

Carrasco, I. \& Castaño, M. (2012). La nueva economía institucional. ICE: Revista de Economía, (865), 43-54.

Frankel, J. \& Romer, D. (1999). Does Trade Cause Growth? The American Economic Review, 8(3), 379-399.

Hall, R. E. \& Jones, C. I. (1999). Why Do Some Countries Produce So Much More Output Per Worker Than Others? The Quarterly Journal of Economics, 114(1), 83-116. Recuperado el 16 de septiembre de 2008, de http://www.jstor.org/stable/2586948

Kaufmann, D., Kraay, A. \& Mastruzzi, M. (2010). The Worldwide Governance Indicators: Methodology and Analytical Issues. Washington: The World Bank.

Kaufman, D., Kraay, A. \& Mastruzzi, M. (2009). Governance Matters VIII: Aggregate and Individual Governance Indicators 1996-2008. Washington: The World Bank.

Kaufman, D., Kraay, A. \& Zoido-Lobatón, P. (1999). Governance Matters. Washington: World Bank Institute-The World Bank.

North, D. (1993). Instituciones, cambio institucional y desempeño económico. México: Fondo de Cultura Económica.

Parker, N., Berthin, G., De Michelle, R. \& Mizrahi, Y. (2004). La corrupción en América Latina: estudio analítico basado en una revisión bibliográfica y entrevistas. Washington: Casals \& Associates/UsAID.

Przeworski, A. \& Curvale, C. (2006). ¿Explica la política la brecha económica entre Estados Unidos y América Latina? En Fukuyama, F. (Ed.). La brecha entre América Latina y Estados Unidos: Determinantes políticos e institucionales del desarrollo económico (pp. 129-166). Buenos Aires: Fondo de Cultura Económica/Fundación Grupo Mayan. 
Raudenbush, S. W. \& Bryk, A. S. (2002). Hierarchical Linear Models: Applications and Data Analysis Methods (2a. ed.). Thousand Oaks: Sage Publications.

Rodrik, D. \& Subramanian, A. (2003). La primacía de las instituciones (y lo que implica). Finanzas y Desarrollo, 40(2), 31-34.

Rodrik, D., Subramanian, A. \& Trebbi, F. (2002). Institutions Rule: The Primacy of Institutions Over Geography and Integration in Economic Development. NBER Working paper $\mathrm{N}^{\circ} 9305$. Cambridge: National Bureau of Economic Research.

Sachs, J. D. \& Vial, J. (2002). Can Latin America Compete? The Latin American Competitiveness Report 2001-2002. Oxford University Press: World Economic Forum.

Sachs, J. D. \& Warner, A. M. (1995). Natural Resource Abundance and Economic Growth. NBER Working paper 5398. Cambridge: National Bureau of Economic Research.

Sen, A. (2015). La idea de la justicia. México: Taurus.

Snijders, T. \& Bosker, R. (2012). Multilevel Analysis: An Introduction to Basic and Advanced Multilevel Modeling. Thousand Oaks: SAge Publications.

Todaro, M. \& Smith, S. (2003). Economic Development. Nueva York, Addison Wesley.

Weil, D. (2006). Crecimiento económico. Madrid: Pearson Educación.

World Economic Forum. (2002). The Latin American Competitiveness (Report 2001-2002). Londres: Oxford University Press.

Recibo el 18 de octubre de 2016. Aceptado el 18 de julio de 2017. 